\title{
Complementación de la Ley 026 para evitar la vulneración de los Derechos Políticos de las Mujeres
}

\author{
Complementation of Law 026 to prevent the violation of the Political Rights of Women
}

Complementação da Lei 026 para prevenir a violação dos Direitos Políticos da Mulher

Artículo recibido en enero 2019

Arbitrado en marzo 2019

Publicado en abril 2019

\section{Alam Patrick Ramos Soliz \\ pramos754@gmail.com \\ ORCID: 0000-0003-0069-0120}

Universidad Privada "Domingo Savio", Bolivia
RESUMEN

ABSTRACT

The unequal political participation of women in Bolivia has generated consequences for society. The objective of this study was to propose the complementation of the Electoral Regime Law in subsection a) of article 11 on gender equality in national elections for the presidency and vice-presidency. The methodology that was used was qualitative and quantitative, the design was descriptive, explanatory of the documentary and field type, I used the analysis-synthesis methods, Induction and Deduction, among others. The technique and instrument used was interview and questionnaire. The population and sample were made up of lawyers and Assembly members, students from the Domingo Savio Potosí Private University, the Tomás Frias Autonomous University and the Valle University. As a result, it was obtained; the proposal for the complementation of Law 026 to avoid the violation of the Political Rights of women. It will allow a breakthrough in Bolivian society.

Key words: Political participation; political rights; gender equality; inequality, law 026
RESUMO

A desigual participação política das mulheres na Bolívia tem gerado consequências para a sociedade. O objetivo deste estudo foi propor a complementação da Lei do Regime Eleitoral na alínea a) do artigo 11. sobre igualdade de género nas eleições nacionais para a presidência e vice-presidência. A metodologia utilizada foi qualitativa e quantitativa, o desenho foi descritivo, explicativo do tipo documental e de campo, utilizei os métodos de análise-síntese, Indução e Dedução, entre outros. A técnica e o instrumento utilizados foram entrevista e questionário. A população e a amostra consistiram em advogados e membros da Assembleia, estudantes da Universidade Privada Domingo Savio Potosí, da Universidade Autônoma Tomás Frias e da Universidade de Valle. Como resultado, foi obtido; a proposta de complementação da Lei 026 para evitar a violação dos Direitos Políticos das mulheres. Isso permitirá um avanço na sociedade boliviana.

Palavras-chave: Política; participação política; direitos políticos; igualdade de gênero; desigualdade, lei 026 


\section{INTRODUCCIÓN}

A pesar de los años de luchas y los avances logrados, la participación política de las mujeres en los procesos de decisión del país, continúa estando pendiente en los debates sobre la calidad de la democracia. Siguen y suman las resistencias a la temática de género, a las cuotas de participación y al acceso equitativo y con alternancia de género en la toma de decisiones.

Por lo tanto, hoy en día, no se puede ignorar que incluir la visión de las mujeres en el escenario político y garantizar la participación, son condiciones sin las cuales no se puede desarrollar una verdadera democracia. Para los movimientos organizados de mujeres, hablar de democracia, requiere mucho más que el reconocimiento del déficit histórico de participación de las mujeres.

Así que, este hecho es reconocido por el Estado Plurinacional de Bolivia y se platea en la nueva Constitución Política del Estado, donde se establece importantes avances para garantizar la inclusión real de las mujeres en los espacios de decisión y los órganos de poder. En este entendido, los cambios producidos en el nivel constitucional contemplan el desafío de garantizar mayor inclusión de los sectores que tradicionalmente estuvieron excluidos, fundamentalmente mujeres e indígenas, a través de la promulgación de leyes que garanticen el ejercicio de los derechos políticos de las mujeres.

De este modo, los Estados están obligados a garantizar el acceso a la justicia de las mujeres, ello constituye un derecho principal que nace en una de las fundamentales obligaciones del Estado que es la de atender al ciudadano o ciudadana que recurre a los órganos jurisdiccionales para que protejan sus derechos vulnerados o amenazados.

Así mismo, se busca transformar la institucionalidad democrática boliviana, el cual promete importantes cambios en la configuración de los órganos de poder, y en la forma de participación de las mujeres en la candidatura para presidencia o vicepresidencia.

Por lo tanto, para el estudio se toma en cuenta la experiencia de diversas expresiones de expertos, profesionales y del movimiento de mujeres bolivianas para demostrar la falta de alternancia en los puestos de presidencia y vicepresidencia del Estado Plurinacional de Bolivia. Partiendo de la incógnita del ¿Cómo evitar la vulneración de los Derechos Políticos de las mujeres establecidos en la Constitución Política del Estado?

Así que, esta investigación tuvo como objetivo central proponer la complementación de la Ley del Régimen Electoral en el Inciso a) del Artículo 11 sobre la equidad, paridad y alternancia de género en las elecciones nacionales para la presidencia y vicepresidencia. Con la finalidad de orientar la solución del problema científico. 
El estudio contribuye como un aporte significativo a la legislación boliviana, a fin de garantizar la igual de derechos políticos de las mujeres. Además, con esta reforma de ley se podrá dar un avance en la sociedad boliviana.

\section{Marco histórico}

\section{Derechos Humanos}

Los derechos humanos según Sacoto (2008) "son garantías universales que protegen a los individuos y grupos frente a acciones $u$ omisiones que puedan afectar sus libertades y su dignidad humana" (p.25). Tiene por objetivo proteger los derechos humanos y las libertades fundamentales inherentes a todas las personas, protección del derecho de las mujeres a la participación política con equidad y alternancia de género.

De acuerdo a la declaración universal de los derechos humanos todos los seres humanos nacen libres e iguales en dignidad y derechos, los cuales deben de ser respetados sin distinción ninguna con base en el sexo, o cualquier otra condición. Asimismo, toda persona tiene derecho a participar en el gobierno de su país, directamente o por medio de representantes libremente escogidos y al acceso, en condiciones de igualdad, a las funciones públicas de su país.

En este sentido, todos son iguales ante la ley y tienen, sin distinción, derecho a igual protección de la ley, así como derecho a igual protección contra toda discriminación que infrinja esta Declaración. Este estudio respalda demandas y acciones de control social hacia la creación e implementación de leyes y mecanismos efectivos para garantizar la inclusión de las mujeres en la política a demás su participación en equidad en los diferentes niveles de poder.

\section{La convención americana sobre los Derechos Humanos}

Los Estados se comprometen a respetar y garantizar el pleno ejercicio de los derechos y libertades reconocidos a toda persona que esté sujeta a su jurisdicción, sin discriminación alguna por motivos de sexo o cualquier otra condición social. Entre otros, todos los ciudadanos deben gozar de los derechos de participar en la dirección de los asuntos públicos, directamente o por medio de representantes libremente elegidos; de votar y ser elegidos en elecciones periódicas, realizadas por sufragio universal e igual y por voto secreto que garantice la libre expresión de electores, y de tener acceso, en condiciones generales de igualdad, a las funciones públicas de su país. -

Otros derechos reconocidos importantes para ejercicio de la participación: a la libertad y la seguridad personal, a que se respete la integridad física, psíquica y 
moral, al respeto a su honra y al reconocimiento de su dignidad, a la libertad de pensamiento, de expresión y de reunión pacífica a asociarse libremente con fines ideológicos, políticos, económicos, laborales, sociales, culturales, o de cualquiera otra índole.

Por lo tanto, en la Convención Americana sobre los Derechos Humanos en su artículo 29c señala que ninguna disposición de esta convención, puede ser interpretada en el sentido de excluir otros derechos y garantías que son inherentes al ser humano o que se derivan de la forma democrática representativa de gobierno.

\section{Derecho de la mujer}

En Bolivia desde la inauguración del período republicano en 1825 hasta la aprobación de la Constitución, mediante Referéndum del 25 de enero de 2009, se reformula por última vez el contenido del texto constitucional, donde se reflejan los avances sobre la inclusión de las mujeres en la participación, además la exigibilidad del cumplimiento de los derechos ciudadanos en el ámbito político, se ha ido dando de forma secuencial

Para Sánchez en Medina (2010), sostiene que: la política constituye uno de los ámbitos sustantivos en el que se expresa la situación de desigualdad entre mujeres y hombres. Sus dispositivos y estructuras restringen el derecho de las mujeres para acceder y participar de la misma manera que los hombres en los espacios políticos y de toma de decisiones $y$, en general, en todos aquellos ámbitos clave de poder determinantes en la definición del interés colectivo de la sociedad.

El sello masculino del proyecto ilustrado no permitió la inclusión de las mujeres en el pacto social, de tal manera Medina (2010) señala que "la Declaración de los Derechos del Hombre y del Ciudadano (1789) reafirmó la exclusión de las mujeres como titulares de derechos, negándoles la categoría de la ciudadanía y, por ende, los derechos inherentes a dicha condición"(p.15).

En este sentido, la filosofía de la llustración puso en entredicho la calidad de humanas de las mujeres, donde expresaban que eran seres cuya "minoría moral" les impedía formar parte de la ciudadanía y, por tanto, ser sujetos.

Por lo tanto, con estos supuestos se negó la capacidad de las mujeres para ejercer autoridad y liderazgo, así como para tomar decisiones vitales en los asuntos considerados del interés colectivo. Medina (2010) indica que "Al excluirlas del estatus de individuos, se les privó de la participación en el mundo público de la igualdad, el consenso, la convención y el pacto social"(p. 16). Todo ello se tradujo en múltiples dimensiones de desigualdad y expresiones de discriminación hacia las mujeres en el acceso, uso y control de oportunidades, adopción de decisiones, manejo de recursos y de servicios, así como en la falta del reconocimiento de sus derechos. 


\section{Participación política de las mujeres}

La política es una actividad orientada en forma ideológica a la toma de decisiones de un grupo para alcanzar ciertos objetivos. También puede definirse como una manera de ejercer el poder con la intención de resolver o minimizar el choque entre los intereses encontrados que se producen dentro de una sociedad. La utilización del término ganó popularidad en el siglo $\mathrm{V}$ a.c cuando Aristóteles desarrolló su obra titulada justamente "Política" (Donoso, 2007).

La participación política es el conjunto de acciones llevadas a cabo por los ciudadanos que no están necesariamente involucrados en la política de forma directa, y cuya acción pretende influir en el proceso político y en el resultado del mismo. El político, por tanto, es aquel que resuelve los conflictos mediante el manejo de voluntades para lograr alcanzar un bien común para todos. Dentro de este contexto, la participación política y la participación ciudadana se encuentran relacionadas (PNUD, 2006).

Inclusive, la desigualdad en la participación política de las mujeres ha motivado, desde las últimas décadas del siglo $\mathrm{XX}$, el cuestionamiento internacional acerca del carácter representativo de los gobiernos, así como el reconocimiento de la necesidad de implementar mecanismos que garanticen a mujeres y hombres las mismas oportunidades de participar en la política de forma efectiva y equilibrada a fin de integrar de igual manera en la agenda pública sus visiones, necesidades, intereses y problemas (PNUD, 2006).

De esta manera, Medina (2010) afirma que la inclusión equilibrada de mujeres y hombres en todos los ámbitos de la sociedad es un medio para garantizar la igualdad de género, a fin de que los diferentes intereses, aspiraciones y necesidades de unas y otros se consideren, valoren y promuevan de igual manera, en términos de derechos, beneficios, obligaciones y oportunidades para, de esta forma, contribuir a mejorar la calidad de la democracia

Este impulso internacional ha motivado la puesta en marcha de políticas de equidad, también conocidas como de la diferenciación para la igualdad (Camacho, 1998). Para Medina (2010) el objetivo "es disminuir y, paulatinamente, cerrar las brechas de desigualdad entre mujeres y hombres a través del fortalecimiento de la ciudadanía femenina, a fin de avanzar hacia la igualdad de género" (p. 20).

Por lo tanto, Medina (2010) señala que el derecho de las mujeres a participar en el ámbito de la política, es reconocido en la actualidad como un derecho humano fundamental, y es requisito básico para la igualdad entre mujeres y hombres, el respeto pleno a la ciudadanía y el fortalecimiento de la democracia (p. 29).

El carácter universal, inalienable e indivisible de los derechos humanos ha posibilitado la definición de la participación equilibrada entre mujeres y hombres en el ámbito político como un tema importante en la agenda internacional de los 
derechos humanos. Sin embargo, tal como apuntan Facio (1992) y Camacho (1998), habría que tener presente que la concepción de los derechos humanos ha variado históricamente de acuerdo con el sentido, valor o contenido que cada época y cada sociedad le han dado; por ello, el reconocimiento de la necesidad de garantizar la participación política equilibrada entre mujeres y hombres ha implicado una serie de procesos históricos de movilización social que ha tenido como protagonistas a las mujeres en su exigencia por lograr el reconocimiento pleno de su ciudadanía. Medina (2010)

Entonces, la creencia de que las mujeres no son tan humanas como los hombres al no poseerlas mismas cualidades, es la justificación para su exclusión del ámbito de la política. Con el tiempo dicha exclusión dio pie a la realización de múltiples movilizaciones femeninas, así como al desarrollo de una mayor conciencia internacional en torno a los derechos de las mujeres, hasta lograr el reconocimiento de que los derechos de las mujeres son también derechos humanos $y$, por ende, requieren estar plenamente integrados en los instrumentos internacionales de la materia. Medina (2010)

Según Facio (2000) señala que:

Este proceso es fundamental porque da cuenta de las situaciones y fenómenos socio históricos que han posibilitado traspasar del reconocimiento de "los Derechos del Hombre" al paradigma de los "Derechos Humanos", tránsito que ha implicado la incorporación de las mujeres como género en lo humano, reconociéndose que son tan humanas como los hombres $y$, por tanto, la discriminación contra las mujeres, por su condición de género, es una violación a los derechos humanos (p. 22).

De acuerdo a Facio (2000), este reconocimiento implica que la realización de la promesa de los derechos humanos depende de que a las mujeres se les respeten sus derechos y ello demanda que la humanidad de las mujeres forme parte de la conciencia colectiva.

\section{MÉTODO}

Se desarrolló bajo el enfoque cualitativo y cuantitativo, el primero, porque trató de determinar la fuerza de asociación o correlación entre dos variables determinados con la finalidad de realizar la generalización y objetivación de los resultados a través de una muestra para hacer una inferencia a una población de la cual procede la muestra. El segundo, porque permitió conocer el fenómeno social relacionado con la doctrina correspondiente a los derechos de las mujeres como un problema social jurídico. 
De esta manera el tipo de investigación que se usó para el estudio fue descriptivo y explicativo; ya que permitió realizar un registro detallado del comportamiento específico de las dos variables identificadas: La incorporación de un inciso al artículo 11 de la Ley del Régimen Electoral y la otra variable, evitar la vulneración de los Derechos Políticos de las Mujeres.

Además el diseño de la investigación de acuerdo con el problema planteado y atendiendo al objetivo general propuesto, fue de tipo documental y de campo, la primera de ella, porque existió la necesidad en la investigación de la vulneración de los derechos Políticos de las Mujeres y la complementación del Inciso a) del Artículo 11 de la Ley Del Régimen Electoral, haciendo consultas a trabajos previos relacionados con el estudio. La segunda se deriva por tratarse de la recolección de datos e información del Tribunal Electoral Departamental que fue recogida en forma directa de la realidad.

A su vez, se desarrollaron los diferentes métodos al momento de realizar el estudio. Entre ellos se tiene los siguientes:

- Análisis - Síntesis, con la finalidad de estructurar los componentes de la complementación al inciso a) del Artículo 11 de la Ley 026, se utilizó el estudio de la literatura consultada y la interpretación de los datos empíricos. Así como para arribar a conclusiones objetivas a partir de fuentes bibliográficas y sistematizar las características de la propuesta referidas a la vulneración de los derechos políticos de las mujeres;

- Inducción y Deducción, porque ayudó a la complementación al inciso a) del artículo 11 sobre la alternancia de género en las listas de candidatos y candidatas a presidencia y vicepresidencia en la ley 026 a partir de la muestra determinada;

- Histórico - Lógico, con ellas determinó la trayectoria de los hechos ocurridos con referencia a la vulneración de los derechos políticos de las mujeres. Además se utilizará este método para precisar los antecedentes y trayectoria de la Ley 026 Ley del Régimen Electoral, referido a la alternancia de género en las listas de candidatas y candidatos a presidencia y vicepresidencia para evitar la vulneración de los derechos políticos de las mujeres a la participación equitativa;

- Análisis Documental, con ella se elaboró las bases teóricas del proyecto de investigación a partir de la sistematización de los elementos aportados por la investigación bibliográfica a realizar con referencia a la complementación de un 
artículo a la Ley 026, para evitar la vulneración de los derechos políticos de las mujeres.

La entrevista y el instrumento fue el cuestionario mediante la ficha de contenido del Tribunal Electoral Departamental de Potosí, el cual, sirvió como una guía para la entrevista, y el cuestionario.

Por otro lado, la población estuvo conformada por 150 profesionales Abogados del Distrito Judicial de Potosí. Asambleístas Departamentales, Estudiantes del último año de la carrera de Derecho de la Universidad Privada Domingo Savio Potosí, Universidad Autónoma Tomás Frías y la Universidad del Valle "Univalle" de la ciudad de Sucre- Bolivia. Y la muestra fue distribuida por el defensor del Pueblo del Estado Plurinacional de Bolivia, la presidenta del Tribunal Electoral del Estado Plurinacional de Bolivia, el Vocal del Tribunal Departamental de Potosí, y las personas con conocimiento del tema.

\section{RESULTADOS Y DISCUSIÓN}

\section{Marco Jurídico Internacional sobre los Derechos Políticos de las Mujeres}

Entre los instrumentos específicos en el ámbito internacional en los que se reconocen y salvaguardan los derechos políticos de las mujeres, se debe hacer mención de la Convención sobre los Derechos Políticos de la Mujer, abierta a la firma por la Asamblea General de la ONU, en su resolución 640 (VII) del 20 de diciembre de 1952.

\section{Instrumentos Jurídicos Regionales}

- Convención Interamericana Sobre Concesión de Derechos Políticos a la Mujer.

- Convención Americana sobre Derechos Humanos (Pacto de San José)

- Convención Interamericana para Prevenir, Sancionar y Erradicar la Violencia contra la Mujer (Belém do Pará)

- Comisión Interamericana de Mujeres (CIM)

\section{Instrumentos Jurídicos Internacionales}

- Protocolo Facultativo de la Convención sobre la eliminación de todas las formas de discriminación contra la mujer CEDAW (1999).

- Declaración y Plataforma de Acción de Beijing (1995)

- Convención Interamericana de la Organización de los Estados Americanos para la Prevención, Sanción y Erradicación de la Violencia contra la Mujer (1994) 
- Convención sobre la Eliminación de todas las Formas de Discriminación contra la Mujer - CEDAW (1979)

- Convención Americana sobre Derechos Humanos (1969)

- Pacto Internacional de Derechos Civiles y Políticos (1966)

- Convención Internacional sobre la Eliminación de todas las Formas de Discriminación Racial (1965)

- Convención sobre los derechos civiles y políticos de la mujer (1953)

- Declaración Universal de Derechos Humanos (1948)

\section{Instrumentos Políticos Internacionales}

- Declaración Universal de Derechos Humanos

- Declaración para la Eliminación de la Discriminación en contra de la Mujer

- Conferencia Mundial de Derechos Humanos

Por lo tanto Archenti (2011) lo ha descrito como un avance de equidad entre los sexos. De esta forma, las políticas públicas de género que impulsan en diferentes esferas la autonomía de las mujeres, y constituyen medidas importantes para la generación de condiciones. El sufragismo se alimentó del cuestionamiento al carácter representativo de los gobiernos y desplegó una movilización cívica para reivindicar la igualdad política entre mujeres y hombres. A través de este movimiento, se afirmó la necesidad de fortalecer los procesos de individuación de las mujeres y de su autonomía en la toma de decisiones vitales en tanto sujetas de derechos.

Aunado a ello, el reconocimiento internacional expresó de cierta manera los avances que estaban logrando las mujeres en el interior de sus países para que fuesen reconocidos sus derechos políticos. Procesos que, han sido diferentes en cada país; aunque la tendencia en la mayoría de las naciones ha sido el reconocimiento jurídico-legal de la igualdad entre mujeres y hombres (igualdad de jure), el cual supone que todas las personas son individuos iguales ante la ley, con la misma capacidad para ejercer sus derechos, lo que ha significado para las mujeres el reconocimiento formal a su estatus de ciudadanas.

Situación actual de la vulneración de los Derechos Políticos de las Mujeres respecto a la falta de alternancia de Género en las listas de candidatos y candidatas a Presidencia y Vicepresidencia.

Partiendo de los resultados obtenidos en la encuesta diseñada para 26 asambleísta y 150 abogados se dieron las respuestas al objetivo central de la investigación. 
En primer lugar, se encontró que la Ley No. 026 no cumple con lo establecido en el marco de la democracia intercultural y equivalencia de condiciones entre mujeres y hombres generando de esta manera la desigualdad entre hombres y mujeres en las elecciones para presidente y vicepresidente del Estado Plurinacional de Bolivia.

En segundo lugar, las personas participantes en la encuesta señalaron que es importante la participación de la mujer en el cargo de Presidenta o Vicepresidenta del Estado Plurinacional de Bolivia. De esta manera, para la sociedad boliviana, la participación de las mujeres en las elecciones nacionales, permitirá prevalecer sus derechos políticos a la equidad paridad y alternancia de género. Ya que la participación social y política de las mujeres ha sido, y es considerada, como una estrategia central en la construcción de la equidad de género y en la profundización de la democracia.

En tercer lugar, tanto asambleísta como abogados, afirmaron que se vulneran los derechos políticos de las mujeres respecto a la alternancia de género en las elecciones para presidenta y vicepresidenta del Estado Plurinacional de Bolivia, Se señaló que toda violación a los derechos humanos puede ser motivo de una interposición de denuncia o queja de violación ante la Comisión Interamericana de incurrir en violaciones a los convenios y pactos internacionales que han sido ratificados por el Estado boliviano. Tras esta situación es necesario proteger y sobre todo prevenir que se vulnere sus derechos de las mujeres.

A pesar de aquello, se reveló que la población boliviana estuvo de acuerdo que exista una representación ya sea en la presidencia o vicepresidencia del Estado, donde no se vulnere los derechos políticos de las mujeres en las candidaturas nacionales para las elecciones presidenciales.

En cuarto lugar, se reveló que existe discriminación hacia las mujeres para poder ser electas como presidentas y vicepresidentas del Estado Plurinacional de Bolivia. En este sentido, la discriminación hacia las mujeres está vinculado por la misma sociedad donde se demuestra un alto índice de discriminación hacia los derechos políticos de las mujeres.

De esta manera, se elaboró el proyecto para la complementación de la alternancia de género al inciso a) artículo 11 de la ley 026, la cual, es considerada sólo una parte de una estrategia más integral dirigida a mejorar la representación femenina y de esta manera dar obligatorio cumplimiento a la Ley 018 Ley del Órgano Electoral Plurinacional; dado a que las mujeres necesitan tener un protagonismo activo y eficaz dentro de sus propios partidos y en la sociedad en su conjunto. La complementación de este inciso a la Ley del Régimen Electoral es esencial para que las mujeres sean tomadas en cuenta por sus partidos políticos para cargos de elección ya sea de presidenta o vicepresidenta, además para que defiendan los derechos humanos, la democracia, representar las demandas de las mujeres 
bolivianas en el escenario político y así poder alcázar la equidad de género en todos los ámbitos de la sociedad.

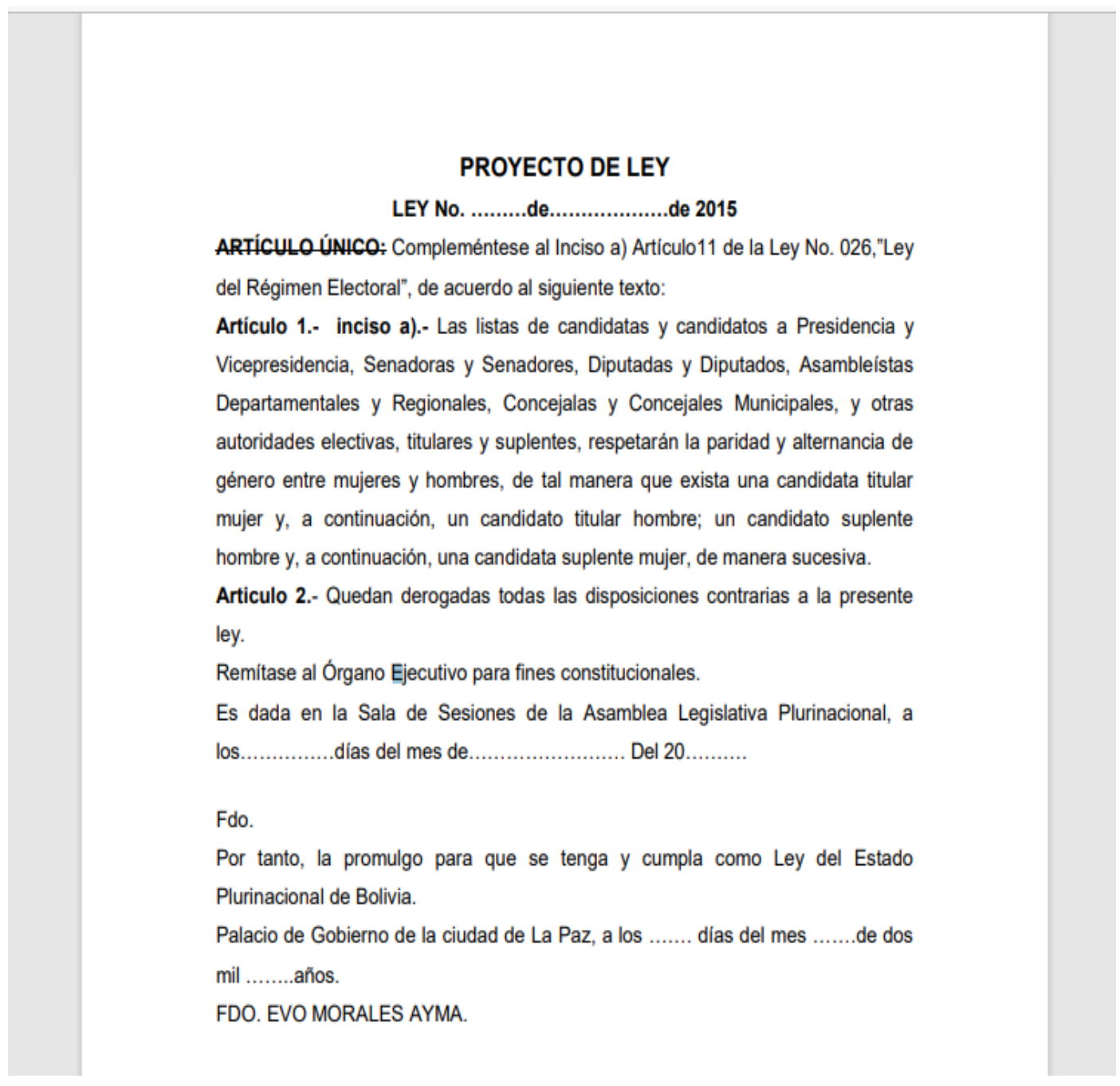

\section{CONCLUSIONES}

Se pudo establecer que si bien se sabe que los derechos políticos de las mujeres están protegidos y garantizados por normativa jurídica nacional e internacional, se pudo observar que la vulneración de sus derechos sigue dando de qué hablar, dejando de lado su dignidad como persona humana. Velando por los derechos políticos de las mujeres, una gran cantidad de normativa nacional e internacional que protegen los derechos de las mujeres, ese conjunto de instrumentos jurídicos tienen propósito que es la igualdad, equidad, alternancia de género en todos los cargos públicos, como la protección de todos sus derechos políticos, garantizar una democracia justa para todos y todas en el bien de la sociedad de los derechos políticos que en ella se enuncian y consagran a favor de toda la humanidad. 
Con el estudio se evidenció que las mujeres sufren vulneración hacia sus derechos políticos, por diversos factores, no tener leyes claras y bien definidas por parte de instancias nacionales. Esta situación, que es incomprensible, debe ser visibilizada de modo que el Estado asuma tareas al respecto, lo que implica entablar un diálogo más sostenido con las instancias que le corresponden para observar los vacíos existentes en este problema, su déficit y sus potencialidades y de esta manera articular en su trabajo el rol ya asumido por diferentes Estados.

Para finalizar, se sustentó que el Estado Plurinacional de Bolivia, debe aprobar un proyecto de ley para garantizar los derechos políticos de las mujeres. y garantizar la alternancia de género en la elección de candidatos a presidencia y vicepresidencia del Estado Plurinacional de Bolivia, de esta manera, se presentó el proyecto de ley, la cual detalla todo lo necesario para que los derechos de las mujeres se respeten, a pesar de las vulneraciones que existen por parte del Estado boliviano, logrando de esta forma el cumplimiento del objetivo de la investigación.

\section{REFERENCIAS}

Donoso, A. (2007). Participación Política de las Mujeres. Santiago-Chile. Edición Asociación latinoamericanas de Organizaciones de Promoción al Desarrollo "ALOP"

Facio, A. (2000). Feminismo, Genero y Patriarcado. Academia, Revista sobre Enseñanza de Derechos de Buenos Aires Año 3, número 6

Facio, A. (1992). Cuando el género suena cambios trae (una metodología para el análisis de género del fenómeno legal) / Alda Facio Montejo. - - 1a. ed. - - San José, C.R.: ILANUD, p.156

Camacho, R. (1998). Sintonizando la conciencia, el voto y los puestos de decisión. Las mujeres y la política en Costa Rica. San José: Fundación Arias para la Paz y el Progreso Humano

Ley 026 Ley del Régimen Electoral

Medina, A. ( 2010). La Participación Política de las Mujeres. México D.F.

Programa de las Naciones Unidas para el Desarrollo "PNUD"(2010). Derechos Humanos de las mujeres y Justicia de Género. La Paz, Bolivia

Sacoto, P. (2008). Factores que inciden en la no conformación de las defensorías comunitarias para el cumplimiento. (Tesis inédita de Maestría). Universidad Tecnológica Equinoccial, Ecuador 